Cómo citar este artículo: Marlés-Betancourt, C., Hermosa-Guzmán, D., \& Correa-Cruz, L. (2021). Fomento de la conciencia hídrica en estudiantes universitarios mediante un juego como estrategia didáctica. Rev.investig.desarro.innov., 11 (2), 361-372.

doi: $10.19053 / 20278306 . v 11 . n 2.2021 .12655$

\title{
Fomento de la conciencia hídrica en estudiantes universitarios mediante un juego como estrategia didáctica
}

\author{
Promoting water awareness in university students \\ through a game as a teaching strategy
}

\author{
Claritza Marlés-Betancourt ${ }^{1}$ \\ Dennyse Hermosa-Guzmán ${ }^{2}$ \\ Lucelly Correa-Cruz ${ }^{3}$
}

Recibido: julio 06 de 2020

Aceptado: octubre 27 de 2020

\begin{abstract}
Resumen
El artículo propone una estrategia didáctica mediante el juego cooperativo para favorecer la conciencia hídrica de la población estudiantil de Administración de Empresas, de la Universidad de la Amazonia, en el piedemonte amazónico colombiano. Es una investigación aplicada al ámbito educativo con enfoque descriptivo de corte transversal, que involucra las fases de diagnóstico, aplicación y evaluación de la intervención didáctica. Los resultados arrojan limitaciones de conocimiento ambiental hídrico en los futuros profesionales de la región, que constituyen insumos para el diseño y construcción del juego cooperativo desde la perspectiva sociocultural. La implementación devela las potencialidades del juego como dinamizador del aprendizaje, motivador de cambios de hábitos y prácticas respecto al uso racional del recurso hídrico y el desarrollo de competencias genéricas, tales como: el trabajo en equipo, la comunicación asertiva, el consenso y la toma de decisiones.
\end{abstract}

Palabras clave: educación ambiental, gamificación, juego educativo, recurso hídrico.

\begin{abstract}
The article proposes a didactic strategy through cooperative games to promote the water awareness of the student population of Business Administration, of the University of the Amazon, in the Colombian Amazon foothills. It is a research applied to the educational field with a descriptive, cross-sectional approach, which involves the phases of diagnosis, application and evaluation of the didactic intervention. The results show limitations of water environmental knowledge in the future professionals of the region, which constitute inputs for the design and construction of the cooperative game from the sociocultural perspective. The implementation reveals the potential of the game as a catalyst for learning, a motivator for changes in habits and practices regarding the rational use of water resources and the development of generic skills, such as: teamwork, assertive communication, consensus and decision decision making.
\end{abstract}

Keywords: educational game, environmental education, gamification, water resource.

1 Administradora de Empresas, Doctora en Educación y Cultura Ambiental, Universidad de la Amazonia, Florencia, Colombia. E-mail: c.marles@udla.edu.co

ORCID: 0000-0002-1314-7517

2 Economista, Doctora en Gerencia y Política Educativa, Universidad de la Amazonia, Florencia, Colombia. E-mail: d.hermosa@udla.edu.co ORCID: 0000-0001-6029-8468

3 Administradora de Empresas, Doctora en Educación y Cultura Ambiental, Universidad de la Amazonia, Florencia, Colombia. E-mail: I.correa@udla.edu.co

ORCID: 0000-0002-4781-0305 


\section{Introducción}

Las estrategias didácticas son de gran importancia en los procesos educativos, debido a que constituyen acciones y procedimientos que apoyan los procesos de enseñanza para favorecer el logro de los objetivos de aprendizaje propuestos (ITESM, 2010). Desde esta perspectiva, las estrategias didácticas lúdicas hacen su aporte como innovación educativa en la formación superior, como alternativa que representa la introducción de cambios en las prácticas educativas. Estas estrategias se convierten en una herramienta eficaz para la comprensión de contenidos en un contexto real y participativo, fomentan la adquisición de saberes, posibilitan la formación de estudiantes activos, la generación de conocimiento compartido, desarrollan habilidades comunicativas y cooperativas (Piñeiro \& Costa, 2015).

En la formación del futuro profesional, se debe incluir la comprensión de la complejidad ambiental de manera transversal en los procesos curriculares, para fortalecer actitudes hacia la conservación del medio ambiente. Igualmente, necesaria es la construcción de conciencia hídrica como perfil distintivo de los profesionales del piedemonte amazónico colombiano, territorio con una riqueza biótica e hídrica de importancia estratégica para la región y el país, que debe ser preservada.

Se comprende por conciencia hídrica, al conjunto de: valores, comportamientos, creencias y actitudes compartidas en sociedad por medio de axiomas cimentados en la educación ambiental. Este constructo social, implica un proceso de sensibilización frente a problemáticas ocasionadas por la interacción antrópica con la naturaleza y en particular con el agua. Es decir, se resalta la necesidad de reconocer el impacto ambiental, ocasionado por las acciones humanas y evidenciado en el detrimento de la naturaleza y de lo hídrico.

Acorde con lo expuesto, se propende por "una conciencia colectiva, basada en el sentido de apropiación y pertenencia" (Isaziga, López \& Huertas, 2019), para proteger los espacios naturales, en particular los asociados a fuentes hídricas. Esto implica un compromiso global para acciones que permitan detener, revertir o afrontar el deterioro de la naturaleza. Desde esta perspectiva, se requiere incidir en la relación entre individuo y naturaleza para afrontar la crisis ambiental y su posible e irreversible impacto en la calidad de vida de las generaciones futuras. Sin embargo, la desinformación y bajo nivel de sensibilización sobre el valor del agua como fuente de vida y desarrollo, evidencia el constante deterioro del recurso hídrico, la limitada participación social e institucional en la formulación de soluciones a largo plazo, convirtiendo la educación en la alternativa para la transformación cultural requerida ante la crisis hídrica actual.

El presente artículo evidencia los principales resultados del diseño e implementación del juego cooperativo, denominado: "Recorre y Gestiona el Agua," elaborado para ampliar los conocimientos sobre el recurso hídrico. Se trata de una experiencia de creación en el aula desde la perspectiva sociocultural con el grupo semiIla, y su posterior aplicación en estudiantes de Administración de Empresas de la Universidad de la Amazonia, del municipio de Florencia, departamento del Caquetá.

Para alcanzar el propósito de la investigación, se emplearon las estrategias didácticas, en particular el juego cooperativo como estrategia para fomentar la concienciación hídrica, mejorar los conocimientos sobre este recurso y contribuir al desarrollo de competencias genéricas en el estudiante universitario, motivadas por su naturaleza cooperativa. La construcción del juego se realiza desde la perspectiva de la gamificación educativa, una metodología que representa la posibilidad de transformar el entorno educativo tradicional a un entorno creativo, motivador y eficiente.

\section{Marco teórico y metodología}

\subsection{Gamificación en la educación superior}

En la tarea de incluir innovación y motivación en las clases, la gamificación se convierte en técnica de aprendizaje que permite incluir el juego con 
temáticas de enseñanza que se adapten a las necesidades de aprendizaje. De acuerdo con Ahmed y Sutton (2017), y Casandra (2017), se considera que el uso de la gamificación permite convertir a los juegos en un objetivo de aprendizaje significativo; con el apoyo de materiales didácticos, se producen clases más prácticas y de mayor participación. La gamificación es una metodología inspiradora en el aula que favorece la enseñanza de contenidos, logrando estudiantes más conscientes de dichos contenidos en su proceso formativo (Keeler, 2014; Alsawaier, 2018).

En esta perspectiva, la gamificación se define como la aplicación de técnicas y dinámicas en las que se utiliza el juego para generar goce, transformar actitudes, motivar y activar el aprendizaje (Romero-Rodríguez, Torres-Toukoumidis \& Aguaded, 2017). Cada día, la gamificación gana más importancia en la educación superior, en donde el juego surge como alternativa para complementar el esquema de enseñanza tradicional, sirve como apoyo a nivel cognoscitivo, emocional y social, debido a que explora actividades y plantea objetivos con recompensas inmediatas, involucrando diferentes experiencias emocionales (Lozada \& Betancur, 2016).

La gamificación utiliza premios y reconocimientos por medio de insignias, puntos extras que promueven la participación en la construcción colectiva de conocimiento, despertando un carácter más divertido de experiencias en el aula (Gil \& Prieto, 2019), al crear nuevos niveles para el procesamiento de información y su contextualización (Vélez \& Yaguana, 2019). Por ende, gamificar en el ámbito de la educación superior, implica modificar aspectos curriculares y realizar una propuesta didáctica que utiliza la mecánica del juego, aprovecha sus ventajas como elemento motivador, social e interactivo (Hernández, 2018), porque favorece la retroalimentación, autonomía y evaluación del aprendizaje adquirido de manera inmediata (Gómez, Fragoso, Arias \& Durpan, 2017).

El aprendizaje basado en juegos, comprende el aprendizaje que involucra recursos didácticos como la gamificación, una estrategia para fomentar el mejoramiento de los procesos cognitivos de enseñanza y aprendizaje, al ser considerado una "forma efectiva de que los estudiantes participen en experiencias de aprendizaje activo" (Charlier, Ott, Remmele \& Whitton, 2012). De acuerdo con Borrego et al. (2018), el aprendizaje basado en juegos, permite la asimilación de conceptos y crea la oportunidad de una retroalimentación inmediata de la práctica educativa, siendo aplicable a diferentes áreas curriculares para evidenciar el progreso y las competencias desarrolladas por el estudiante (Bodnar et al., 2016).

La incorporación de juegos en los procesos de enseñanza y aprendizaje es una decisión acerta$\mathrm{da}$, que propende por el logro de conocimientos más sólidos (Álvarez, Cepeda, Alarcón \& López, 2020). El diseño del juego se establece de acuerdo con el objetivo de aprendizaje a lograr (Sosa et al., 2018); de esta manera, permite comprender la complejidad de la teoría y la enseñanza, en donde intervienen múltiples intereses y propuestas de solución (Sosa-Espinosa \& PérezAlonso, 2014).

El elemento principal del aprendizaje basado en juegos, es en esencia el juego como recurso educativo, utilizado desde la antigüedad para el desarrollo de habilidades en niños, jóvenes $y$ adultos. Esto ya que favorece y enriquece los procesos de enseñanza y aprendizaje, que logra integrar elementos como: la participación, el desarrollo creativo en la solución de problemas reales en un contexto socio-ambiental.

En esta perspectiva, se retoma el juego cooperativo, con la característica que todos los participantes deben alcanzar una meta común trabajando juntos, porque en el escenario de juego todos ganan o todos pierden. Como lo expresan Retamal, Saldivia, Luengo y Ramírez (2014), en el juego cooperativo todos los jugadores compiten contra los elementos no humanos; en lugar de competir entre ellos, deben unir esfuerzos y utilizar habilidades individuales para el beneficio colectivo en un ambiente que exige: compartir ideas, confianza, armonía y diálogo. Lo anterior unido a adquirir 
una conciencia solidaria, enfocada en renunciar al individualismo y la posesión exclusiva, para: compartir con el compañero, aunar esfuerzos y cumplir la misión del juego.

El uso del juego cooperativo en el contexto de la educación superior, fomenta un espacio académico con un entorno de aprendizaje participativo, vivencial y dinámico, reflexivo y crítico, orientado a la transformación de grupos en el aula de clase con énfasis de enseñanza social (Agüero, 2019; Cerdas, 2013). Esta metodología de juego favorece la aceptación y respeto de las opiniones de cada participante, ya que se fundamenta en la construcción de acuerdos para dar solución a situaciones específicas (Calvo, 2018). En este sentido, el juego cooperativo representa una alternativa metodológica que contribuye a los procesos de inclusión de la educación ambiental en el contexto universitario, y de manera particular la educación relativa al agua. Esta última se define por el Programa Hidrológico Internacional, como: un tema de importancia mundial y transversal a todas las áreas del desarrollo, que permita generar conocimiento, mejorar las capacidades en materia de hidrología y valores para la protección y manejo sostenible del recurso hídrico (Unesco, 2019).

\subsection{Metodología}

La investigación es aplicada en el ámbito educativo con un enfoque exploratorio descriptivo, de corte transversal. La población objeto de estudio corresponde a estudiantes del ciclo de formación básica y ciclo profesional, del Programa de Administración de Empresas, de la Universidad de la Amazonia, en Colombia.

El diseño metodológico aplicado, consta de tres fases: Fase Diagnóstica (1), Fase Aplicada (2), y Fase Evaluativa (3). La fase 1 se desarrolló con la técnica de grupo focal, con una guía semiestructurada de preguntas, de acuerdo con tres categorías de estudio: importancia del recurso hídrico, problemáticas que afectan el recurso hídrico en la región, conocimientos sobre recurso y gestión hídrica. La fase 2 implica la construcción colectiva del juego cooperativo, con estudiantes del semillero de investigación SIEMA y docente investigador, que comprende el proceso de diseño, desarrollo y validación por pilotaje y comunidad académica. Esta fase también incluye la intervención didáctica, desarrollada a través de secuencias didácticas con 14 sesiones de trabajo presencial. La fase 3 se implementó con la finalidad de identificar el impacto de la intervención didáctica del juego desarrollado, utilizando la técnica de grupo focal, para: (i) determinar la pertinencia del juego en relación a la temática de investigación y (ii) los aspectos claves de la experiencia en la intervención didáctica.

\section{Resultados y discusión}

\subsection{Diseño y construcción del juego}

El proceso de diseño y construcción del Juego: Recorre y Gestiona el Agua (RGA), involucró un diagnóstico para identificar los conocimientos previos de la población estudiantil, como insumo del proceso de diseño y construcción del juego, que consta de cinco etapas: planeación, diseño, desarrollo, pilotaje y revisión. Estas etapas se desarrollaron en una secuencia didáctica de catorce sesiones de trabajo conjunto, entre el grupo semilla y la docente investigadora (Ver figura 1). Una vez culminada la construcción del juego, se realizó la intervención didáctica en la comunidad académica, con el propósito de validar empiricamente la estrategia didáctica diseñada. 


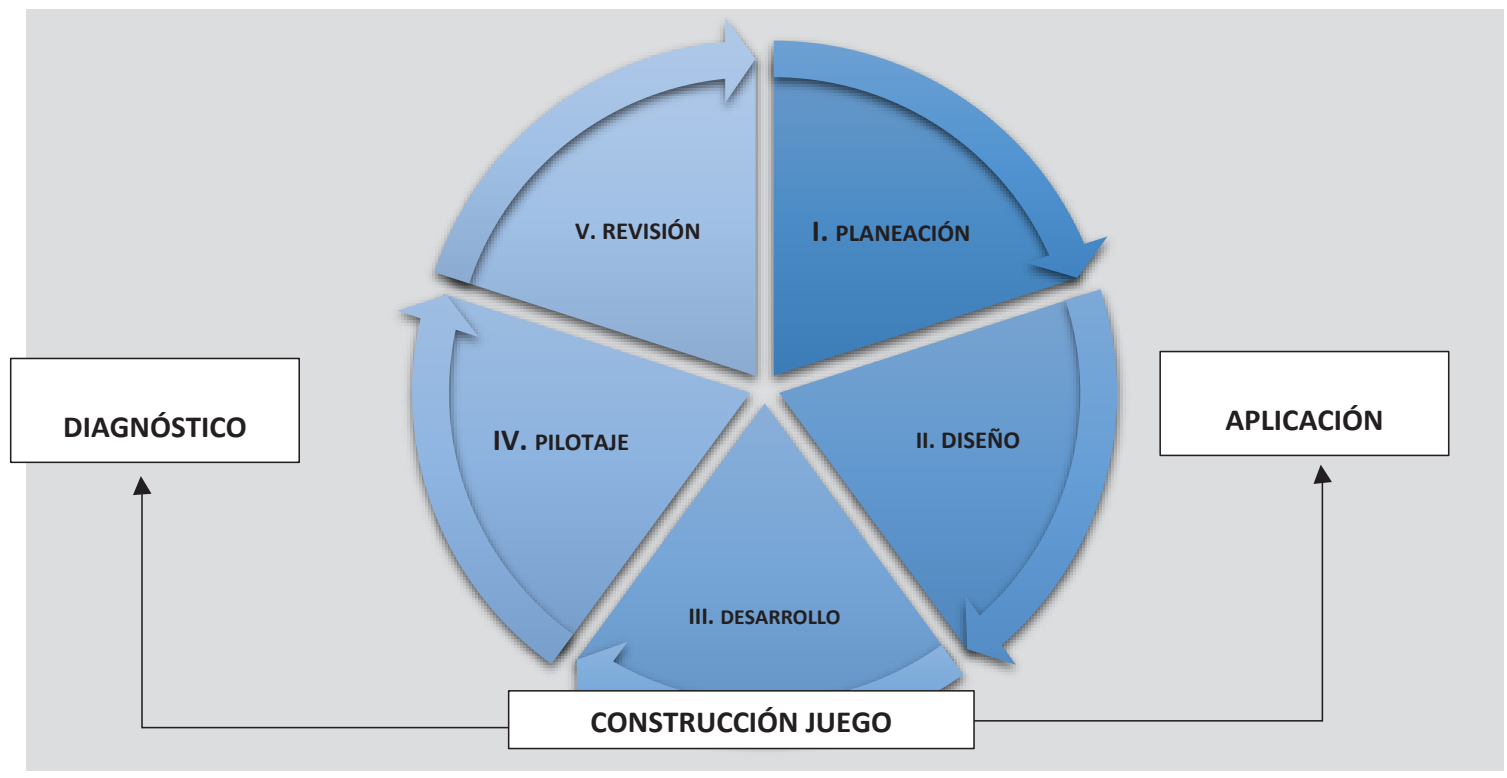

Figura 1. Etapas de la elaboración del Juego.

Los resultados del diagnóstico, evidenciaron el reducido conocimiento de los estudiantes acerca del recurso hídrico y la concepción general de la riqueza hídrica regional, que se refleja en el paradigma de abundancia e inagotabilidad del recurso. Así mismo, los resultados traslucen el escaso interés en conocer y proponer alternativas de conservación del recurso; sin embargo, reconocen las actividades económicas que afectan el recurso en la región, dentro de las cuales no se incluyen las aguas residuales domésticas e industriales.

Es importante mencionar que, al indagar sobre las acciones individuales y grupales realizadas para favorecer la cultura del agua, los estudiantes no identifican actividades económicas y extractivas que están afectando la calidad del recurso. La etapa de planeación, permitió la contextualización y formulación de ideas afirmadas con técnicas de creatividad, para lograr una propuesta de enseñanza-aprendizaje que permita solucionar la falencias identificadas en el diagnóstico.

\section{Principios de diseño del juego}

Para el diseño del juego RGA, se tuvo en cuenta el marco conceptual MDA (Hunicke, LeBlanc \&
Zubek, 2004), que propone pautas de diseño enfocadas en tres ejes, representados en: las Mecánicas (reglas en general), Dinámicas (cómo se desarrollan las reglas en el juego), Estéticas (gráficos, la música, la ambientación, la historia que se cuenta en el juego). La perspectiva de diseño MDA, es un proceso que analiza cada elemento que compone la arquitectura del juego, y los articula con el contexto específico en el que se desarrolla e implementa el juego y el objetivo propuesto (Lombardelli, Torres \& Fernández, 2020).

-Mecánicas y Dinámicas del Juego Recorre y Gestiona el Agua por Colombia. Es un juego cooperativo, en el que los jugadores realizan acciones fundamentadas en la cooperación para alcanzar las metas propuestas, con las características particulares de sus personajes. El juego muestra un recorrido geográfico por las diferentes zonas de Colombia (Estética), y da a conocer la diversidad de problemáticas del recurso hídrico que existen en el país y el departamento del Caquetá (Figura 2). En este caso, cuatro personajes (roles de los jugadores), trabajan conjuntamente para eliminar problemáticas que causan impactos negativos en el recurso hídrico. 




Figura 2. Tablero de Juego (diseño de Jhon Janner Ruiz).

El juego presenta situaciones reales que afectan la calidad, cantidad, disponibilidad y uso del agua, agrupadas en tres categorías. Las categorías hacen referencia a las actividades del sector agropecuario, la deforestación, la minería, las actividades industriales y aspectos culturales y sociales, relacionados con los hábitos y usos que generan problemas ambientales en el recurso hídrico de la realidad colombiana. A continuación, se enlistan las categorías dentro del juego:

i) Ambiental: actividades de deforestación y sistemas de producción agropecuarios.

ii) Minería e hidrocarburos: actividades de minería legal e ilegal, la explotación de hidrocarburos, carbón, oro, níquel y demás materiales no renovables.

iii) Social y cultural: usos y hábitos que afectan el recurso hídrico, escasez y calidad del agua.

Las categorías contienen casos de Problemas actuales del Recurso Hídrico (PRH), representa- dos en cartas de juego. El juego cuenta con 98 cartas en total, de las cuales 39 corresponden a la categoría de ambiental, 37 a minería e hidrocarburos, y 31 a aspectos sociales- culturales. Además, el juego contiene un mazo de cartas con los 32 departamentos de Colombia, divididas en los colores amarillo, azul y rojo, las cuales permiten desplazamientos en el tablero de juego.

Asimismo, se estableció como elemento adicional al tablero de juego una herramienta que mide la cantidad de agua, que refleja cambios a medida que los participantes disminuyen o aumentan los focos de problemas (PRH). La medición puede generar que los participantes pierdan el juego al no realizar trabajo cooperativo y comunitario, para limpiar aquellas zonas más afectadas por las problemáticas ambientales. La figura 3 muestra la perspectiva global de los componentes, dinámicas y mecánicas del juego. 

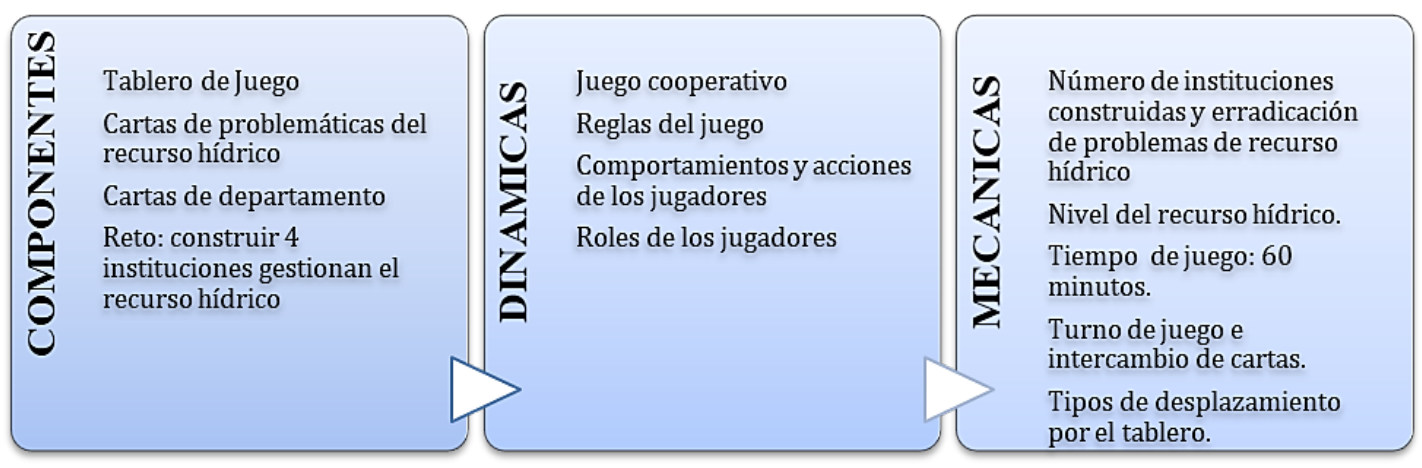

Figura 3. Descripción de las mecánicas y dinámicas del juego RGA.

-Dinámicas. El juego incluye una metodología cooperativa inmersa en el diseño, presente en los componentes y roles de cada jugador: biólogo, científico y ambientalista. Se precisa la integración de todos los participantes para alcanzar la misión del juego, eliminando los problemas ambientales en las diversas regiones del país. En este sentido, cada jugador asume un rol con habilidades especiales para ser utilizadas de manera estratégica durante el juego.

El jugador que asume el rol de Biólogo, puede prevenir los problemas de recurso hídrico para evitar su propagación acelerada; el Ambientalista posee la habilidad de desplazarse directamente por el tablero de juego al lugar deseado, el Científico puede exterminar todos los focos de contaminación si adquiere previamente las cartas del departamento del mismo color; el Gestor podrá ubicar la ficha que cumple la función de instituto investigador, acción que suprime la posibilidad de volver a presentar PRH o focos contaminantes. El juego se gana si logran construir cinco institutos en el tablero de juego; se pierde si el nivel del agua llega a cero. Este nivel disminuye cada vez que se presentan más de siete PRH o contaminación en alguna región, o no se logra la meta de institutos de gestión hídrica construida.

Una vez culminado el diseño, se elaboraron los prototipos del material didáctico (tablero, fichas, tarjetas), la identidad visual, material de apoyo y la estética del juego, según las características del tema. Luego se realizaron los pilotajes, en primera instancia con del grupo semilla y luego con diversos grupos de estudiantes, para evidenciar la utilidad y aplicabilidad de los elementos diseñados. Es decir, se realizó la evaluación del material didáctico, del tiempo de cada una de las acciones, la pertinencia de las reglas, los roles y la relación del juego con el tema de estudio. Lo anterior permitió reajustar algunos aspectos del juego, tales como: la pertinencia de los roles, reglas y materiales empleados.

\subsection{Aplicación del juego RGA}

La intervención didáctica se realizó en tres momentos: Contextualización sobre el recurso hídrico, desde la perspectiva de la glocalidad; explicación detallada del juego cooperativo, el material didáctico del juego, las reglas, los personajes y sus habilidades espaciales, para dar a conocer la importancia del trabajo cooperativo; y los riesgos de no alcanzar la meta establecida. Posteriormente, se define el tiempo para el desarrollo del juego (1 hora), con el apoyo del grupo semilla, el cual realiza orientación y seguimiento durante el tiempo en que los jugadores logran el objetivo (figura 4). Por último, se realizó la premiación de los ganadores y la evaluación de la experiencia. 


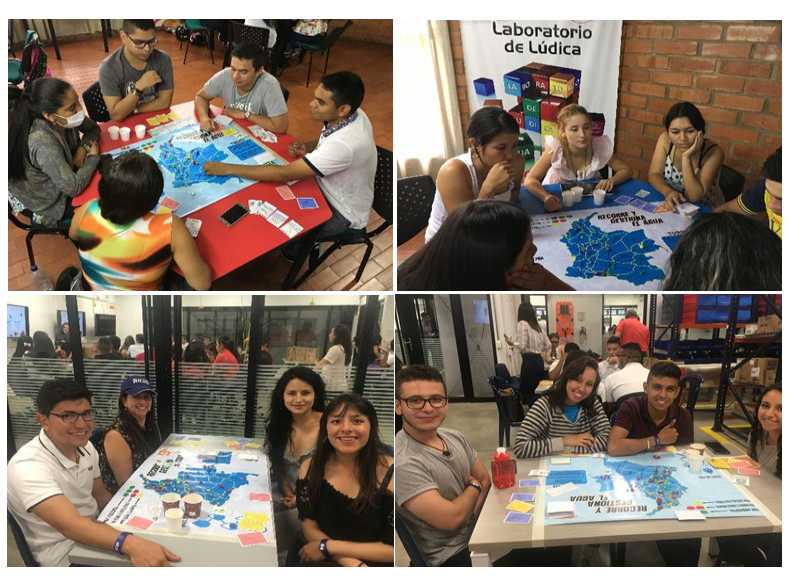

Figura 4. Aplicación del Juego: Recorre y Gestiona el Agua.

La etapa de evaluación y retroalimentación de la experiencia, es de gran relevancia para la investigación. En ella se indaga sobre la experiencia vivida por el estudiante, el mensaje que transmitió el juego y el grado en que se cumplió el objetivo. Lo anterior permitió evidenciar: la pertinencia del material empleado, las mecánicas, las dinámicas de juego en la sensibilización, y la contribución en la concienciación de lo hídrico.

\subsection{Evaluación de la experiencia del juego RGA}

Los resultados del grupo focal, diseñado para valorar la pertinencia del tema del juego en relación a la temática de investigación, y la experiencia del juego, se presenta en las categorías: contenido, experiencia, percepción y aplicación. En ésta última categoría, se destaca el juego como instrumento sensibilizador, que contribuye a mejorar el interés y crear conciencia hacia la conservación de los recursos hídricos y la cultura del agua.

El gráfico de conglomerados (figura 5), muestra la relación entre las categorías identificadas con el análisis de contenido, en el cual destaca la relación entre el conocimiento y la diversión como estrategia de enseñanza y aprendizaje. Igualmente, se resalta la relación entre las estrategias didácticas lúdicas como alternativa para mejorar el aprendizaje. Además, se enfatiza en la importancia del agua, evidenciando que el juego y sus temáticas hídricas contribuyen a concientizar, sobre: actividades, problemas e impactos generados por el hombre; asimismo, destacan la relación directa que existe entre personas y consumo.

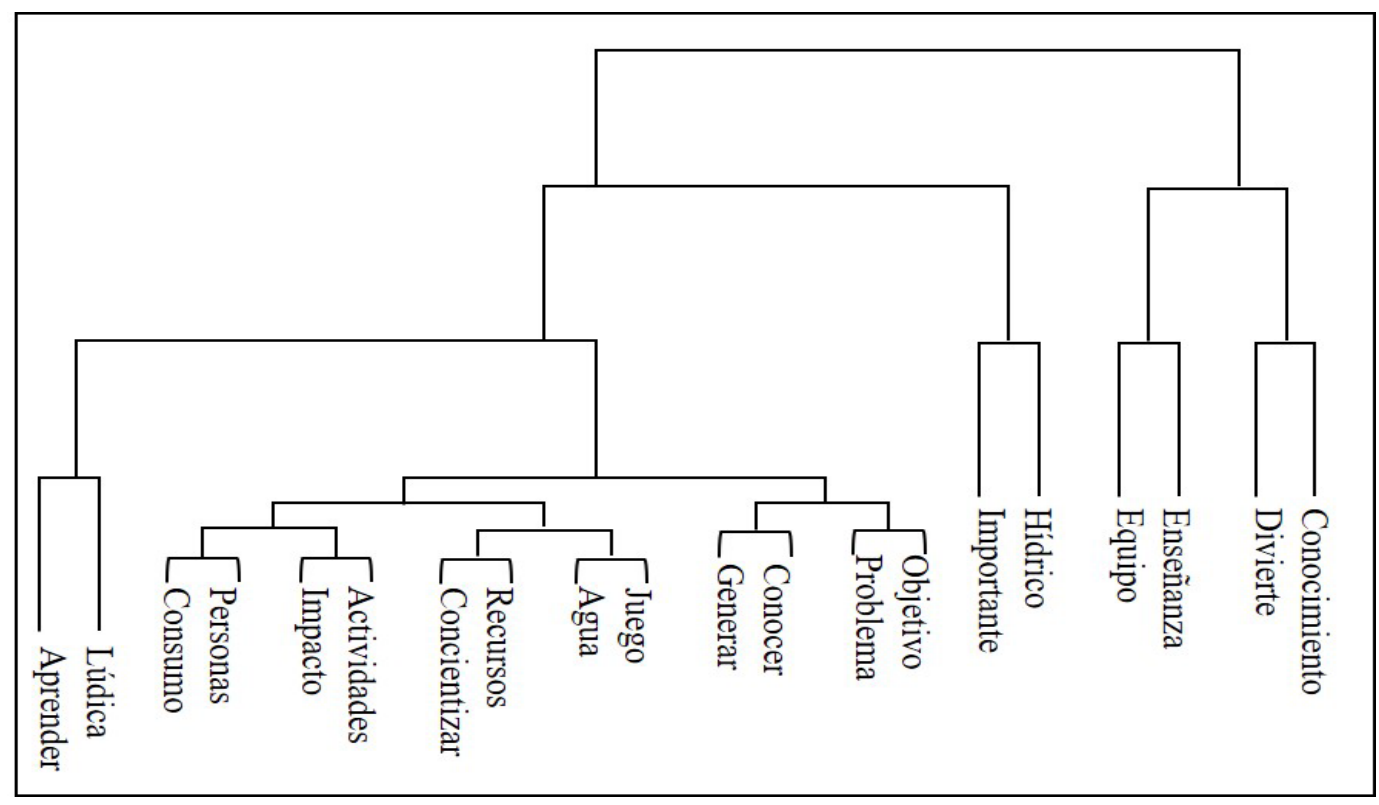

Figura 5. Análisis de Conglomerado. 
En el análisis sobre la utilidad, claridad y practicidad didáctica del juego, los estudiantes reconocen la pertinencia del tema desarrollado. Además, especifican que los componentes empleados en el juego y el material didáctico: despiertan el interés en el tema, permiten adquirir conocimiento sobre el recurso hídrico, el uso, sus problemáticas, y acerca de la importancia de la gestión hídrica en el aporte de soluciones para el beneficio social mayoritario. Es importante destacar que, para los participantes en la intervención didáctica, la información que entrega el juego permite sensibilizar y motivar cambios en los hábitos y prácticas relacionadas con el agua.

El juego permite crear una visión real del recurso hídrico en Colombia y los problemas de gestión que existen, ubicando al estudiante en un contexto social, económico y cultural. Los participantes logran evidenciar la magnitud de las causantes que afectan la calidad, cantidad y disponibilidad del agua en el país. Los estudiantes resaltan la prioridad de empezar por crear conciencia sobre el uso del agua en el núcleo familiar, como mecanismo para motivar acciones colectivas.

Los estudiantes resaltaron de manera reiterativa, el aporte de la metodología cooperativa del juego, debido a que es indispensable la buena comunicación para avanzar. Se considera que el trabajo en equipo y el consenso en la toma de decisiones, permiten cumplir la misión de ganar el juego. También se indica la efectividad del juego para ampliar conocimientos e informar sobre problemas hídricos, por la necesidad de generar conciencia hídrica y motivar transformaciones desde sus hábitos y prácticas, para aportar en los procesos de mitigación y conservación.

Los participantes también señalan la importancia de fortalecer iniciativas fundamentadas en la gamificación, que promuevan acciones para cuidar las fuentes hídricas del departamento. Además, indican la necesidad de incluir la dimensión ambiental en el proceso de formación como Administradores de Empresas, mediante estrategias que aporten en la sostenibilidad de la región.
En este sentido, Gallardo, Olivera y Mexxomo (2019), destacan que la dimensión ambiental y la sostenibilidad, son aspectos que requieren ser incluidos en la formación de profesionales, debido a que existe desinterés del estudiantado en participar de acciones a favor del ambiente, y realizar comportamientos proambientales. Igualmente, De la Rosa, Giménez y Maldonado (2019), consideran que las instituciones educativas deben privilegiar la creación de espacios de aprendizaje creativos y diferentes a los tradicionales, teniendo en cuenta que el estudiante acrecienta su grado de responsabilidad social con alternativas didácticas que contribuyen a transformar la relación del individuo con el entorno socioambiental.

El juego diseñado e implementado, aborda situaciones reales y actuales del contexto regional y nacional, permitiendo la conexión con conocimientos previos sobre el tema de estudio. Al respecto, Gutiérrez (2017), afirma que los contenidos relacionados con casos ambientales, aportan a la comprensión de conocimientos, al establecimiento de relaciones y alternativas inherentes al entorno cercano del estudiante.

\section{Conclusiones}

Inicialmente, se evidenció la percepción de los estudiantes del Programa de Administración de Empresas acerca del recurso hídrico, las acciones que afectan la calidad, cantidad y disponibilidad en la región del Caquetá, y las iniciativas individuales y colectivas para mitigar los impactos de la acción antrópica. El análisis trasluce la limitada existencia de acciones para promover el uso sostenible del recurso y su conservación. El diagnóstico destaca la insuficiente información proporcionada durante la formación profesional de los participantes, sobre el recurso hídrico y su adecuada administración. Esto guarda relación con el desinterés de la población universitaria en: conocer el impacto de las actividades humanas, los procesos de gestión como alternativa de mejoramiento, y en participar en movimientos que propenden por la conservación del recurso hídrico. 
El juego cooperativo: Recorre y Gestiona el Agua por Colombia, genera un cambio a nivel cognoscitivo sobre la importancia del agua para el desarrollo de cualquier actividad productiva y para la satisfacción de necesidades básicas. Esto en la medida que el participante evidencia las problemáticas que asume en el recurso hídrico en Colombia, lo cual despierta el interés y la necesidad de fomentar una consciencia hídrica y transformar prácticas y hábitos.

El implementar la metodología de juego cooperativo, a través del diseño didáctico de la estrategia de enseñanza y aprendizaje, fomentó: la comunicación, la toma de decisiones y el consenso. La información suministrada en el juego, al ser debatida por los participantes, permite ser recordada y compartida en otros ambientes y grupos sociales, transmitiendo un mensaje sensibilizador.

Los resultados permiten pensar más allá de las prácticas pedagógicas que se realizan en la educación superior, e incitan a reflexionar sobre el proceso de enseñanza y aprendizaje y la manera en cómo aplicar los conocimientos en un contexto real. Sumado a ello, surge la inquietud de involucrar la dimensión ambiental en la transversalidad de los contenidos curriculares de los profesionales en Administración de Empresas, para favorecer la inmersión del desarrollo profesional en el entorno socioambiental actual, que permita mejorar el nivel de aprehensión conceptual y configuración de pensamiento crítico. Lo anterior, mediante estrategias didácticas contemporáneas como la lúdica, para promover el desarrollo individual y colectivo de una cultura ambiental.

\section{Agradecimientos}

Este artículo es resultado de la tesis doctoral: el Juego Educativo sobre Gestión Hídrica como Alternativa para la Educación Ambiental en la Universidad de la Amazonia, de la primera autora, presentado al Doctorado en Educación y Cultura Ambiental.

\section{Referencias}

Agüero, E. C. (2019). Reflexiones acerca de prácticas lúdicas en educación en derechos humanos: el juego cooperativo. Universidad en Diálogo: Revista de Extensión, 9(1), 159-170. Recuperado de: https://www.revistas.una.ac.cr/index.php/ dialogo/article/view/11956

Ahmed, A. \& Sutton, M. J. D. (2017). Gamification, serious games, simulations, and immersive learning environments in knowledge management initiatives. World Journal of Science, Technology and Sustainable Development, 14(2/3), 78-83. https://doi.org/10.1108/ WJSTSD-02-2017-0005

Alsawaier, R. S. (2018). The effect of gamification on motivation and engagement. International Journal of Information and Learning Technology, 35(1), 56-79. https://doi.org/10.1108/ IJILT-02-2017-0009

Álvarez, V. M., Cepeda, H. F., Alarcón, E. Y. P., \& López, J. C. (2020). Estrategias lúdicas en el aprendizaje de la nomenclatura química inorgánica. Cátedra, 3 (1), 59-74. Recuperado de: http:// revistadigital.uce.edu.ec/index.php/CATEDRA/ article/view/1966

Bodnar, C. A., Anastasio, D., Enszer, J. A., \& Burkey, D. D. (2016). Engineers at play: Games as teaching tools for undergraduate engineering students. Journal of Engineering Education, 105(1), 147-200. https://doi.org/10.1002/jee.20106

Borrego, C., Fernández, C., Robles, S., y Blanes, I. (2016). Room escape en las aulas: actividades de juegos de escape para facilitar la motivación y el aprendizaje de las ciencias de la computación. Revista del Congreso Internacional de Docencia Universitaria e Innovación, 3, 1-7. Recuperado de: https://www.cidui.org/revistacidui/index.php/ cidui/article/view/851

Calvo, L. (2018). Los juegos cooperativos y el acceso a la información desde la extensión universitaria. E-Ciencias de la Información, 8(1), 119-130. https://doi.org/10.15517/eci. v8i1.30562 
Charlier, N., Ott, M., Remmele, B., \& Whitton, N. (2012). Not just for children: game-based learning for older adults. In 6th European Conference on Games Based Learning, Cork, Ireland. 102-108.

De la Rosa, D., Giménez, P., \& Maldonado, C. (2019). Educación para el desarrollo sostenible: el papel de la universidad en la Agenda 2030. Recuperado de: http://93.174.1.52/handle/10641/1691

Gutiérrez, L. H. (2017). La educación ambiental: una estrategia didáctica para favorecer el conocimiento escolar deseable en educación básica secundaria en la Institución Educativa Departamental Ignacio Pescador de Choachí Cundinamarca (Tesis doctoral). Universidad de la Salle, Bogotá, Colombia. Recuperado de: https://ciencia.lasalle. edu.co/doct_educacion_sociedad/10/

Gallardo, O. A., Olivera, C., \& Mezzomo, F. A. (2019). El estudiantado universitario de UNESPAR-Brasil: sus percepciones sobre el desarrollo sostenible. Revista Educación, 43(1).

Gómez, E., Fragoso-Campón, L., Arias-Trujillo, J., \& Durán-Barroso, P. (2017). Gamificación aplicada a la gestión de recursos hídricos. Innovación Docente en la Ingeniería, 36, 36-41.

Guerrero, L. (2019). Estrategias de gamificación en la universidad: el uso de Class Dojo. In Nuevas tecnologías en el proceso de enseñanza-aprendizaje, 65-70. Universidad Técnica Particular de Loja.

Hernández, I. (2018). El Ministerio de Robin Hood: una experiencia de gamificación. Revista números. Didácticas de las matemáticas, 1(98), 153-162. Recuperado de: http://funes.uniandes. edu.co/12892/

Hunicke, R., LeBlanc, M., \& Zubek, R. (2004). MDA: A formal approach to game design and game research. In Proceedings of the AAAI Workshop on Challenges in Game Al 4, (1), 1722.

Instituto Tecnológico y de Estudios Superiores de Monterrey, ITESM. (2010). Dirección de investigación y desarrollo. Capacitación en estrategias y técnicas didácticas. Recuperado de: http:// sitios.itesm.mx/va/dide/documentos/infdoc/ Est_y_tec.PDF

Isaziga, L. M., López, M. J., \& Huertas, L. A. (2019). Pedagogía de la tierra como elemento dinamizador de la conciencia ambiental (Tesis de maestría). Universidad Cooperativa de Colombia, Bogotá. Recuperado de: https://repository.ucc.edu.co/ handle/20.500.12494/6588

Keeler, A. (2014). Beyond the worksheet: playsheets, GBL, and gamification. Edutopia, 1-3.

Lozada, C., \& Betancur, S. (2017). La gamificación en la educación superior: una revisión sistemática. Revista Ingenierías Universidad de Medellín, 16(31), 97-124.

Lombardelli, M. J., Torres, D., \& Fernández, A. (2020). Motivando la construcción de conocimiento colectivo a través del juego: un análisis de caso implementando medios físicos. Cuadernos del Centro de Estudios en Diseño y Comunicación. Ensayos, (110), 77-91.

Piñeiro, T. \& Costa, C. (2015). ARG (juegos de realidad alternativa). Contribuciones, limitaciones y potencialidades para la docencia universitaria. Comunicar, 44, 141-148.

Quintana, J. G., \& Jurado, E. P. (2019). Juego y gamificación: Innovación educativa en una sociedad en continuo cambio. Revista Ensayos Pedagógicos, 14(1), 91-121.

Romero-Rodríguez, L. M., Torres-Toukoumidis, Á., \& Aguaded, I. (2017). Ludificación y educación para la ciudadanía. Revisión de las experiencias significativas. Educar, 53(1), 109-128.

Retamal, F. C., Saldivia, H. M., Luengo, F. Q., \& Ramírez, G. V. (2014). El juego cooperativo como herramienta de aprendizaje. VIREF, Revista de Educación Física, 3(1), 1-15.

Sosa, A., Gielen, E., Palencia, J. S., Pérez, Y., Moreno, M., Temes, R. \& Trénor, M. (2018). El aprendizaje lúdico como estrategia didáctica: El territorio en Juego. En IN-RED 2018. IV Congreso Nacional de Innovación Educativa y Docencia en 
Red. 1493-1503. Editorial Universidad Politécnica de Valencia.

Sosa-Espinosa, A., \& Perez-Alonso, Y. (2014). "Role playing: Una buena herramienta para aprender". En Martí Selva, ML.; Calafat Marzal, MC. (2014). I Jornadas de Investigación de la Facultad de ADE. Editorial Universidad Politécnica de Valencia.

UNESCO (2019). Programa Hidrológico Intergubernamental. Recuperado de: https://es.unesco. org/themes/garantizar-suministro-agua/hidrologia 